\title{
Relación del tratamiento por dependencia de la cocaína con los valores personales de apertura al cambio y conservación
}

\section{Relationship between Cocaine Dependence Treatment and Personal Values of Openness to Change and Conservation}

| Jesús Salz Galdós*; Isabel Martínez Sánchez ${ }^{\star *}$
*Universidad Complutense de Madrid, Colaborador Honoríico

**Universidad de Castilla-La Mancha, Profesora Contratada Doctora

Enviar correspondencia a:

Jesús Saiz. Despacho 1613, Dpto. de Psicología Social,

Fac. de Ciencias Políticas y Sociología, Campus de Somosaguas.

Universidad Complutense de Madrid. 28223 Madrid.

E-mail: saiz.jesus@gmail.com.

\section{RESUMEN}

Objetivo: Analizar la relación entre la asistencia a un tratamiento de drogodependencia y los valores de Apertura al cambio (Autodirección, Estimulación y Hedonismo) y Conservación (Tradición, Conformidad y Seguridad) de Schwartz en sujetos consumidores de cocaína. Método: El presente estudio, de naturaleza cuasi-experimental, se realizó sobre una muestra de 411 adultos consumidores de cocaina que fueron agrupados en función de si llevaban más de tres meses de tratamiento, menos de tres meses o no recibian ningún tratamiento. A través del cuestionario de valores personales PVQ de Schwartz se midió la prioridad que cada uno de estos grupos de sujetos daba a los valores de Conservación y Apertura al cambio. Resultados: Los sujetos que habian iniciado el tratamiento hacía más de tres meses tendian a puntuar menos en los valores de Apertura al cambio que aquellos sujetos que no estaban bajo tratamiento, aunque no aparecieron diferencias significativas en estos valores entre los sujetos que no estaban bajo tratamiento y los que hacia menos de tres meses que lo habian iniciado. Además, se observó que los sujetos bajo tratamiento, independientemente de que éste fuese inferior o superior a tres meses, puntuaron más alto en los valores de Conservación que los sujetos consumidores de cocaina que no estaban bajo tratamiento. Discusión: Estos resultados ponen de manifiesto la relación de la asistencia a un programa del tratamiento por dependencia de cocaina con los valores personales de los sujetos consumidores y su potencial importancia en el tratamiento y rehabilitación de la dependencia de esta sustancia.

Palabras clave: consumo de cocaina, tratamiento por dependencia a la cocaina, valores de apertura al cambio, valores de conservación.

\section{ABSTRACT}

Objective: Io analyze the relationship between participation in a drugaddiction treatment program and Schwartz's values of Openness to change (Self-direction, Stimulation and Hedonism) and Conservation (Tradition, Conformity and Security) in cocaine users. Method: The present quasi-experimental study was carried out on a sample of 411 adult cocaine users, grouped according to whether they had begun treatment more than three months earlier or less than three months earlier or they were not receiving any treatment. Using Schwartz's Personal Values Questionnaire (PV0), we measured the priority given by each of these groups to the values of Conservation and Openness to change. Results: Participants who had initiated the treatment more than three months earlier tended to score lower in the values of Openness to change than those who were not in treatment, though there were no significant differences in these values between those who were not in treatment and those who had been in treatment for less than three months. On the other hand, it was observed that participants in treatment, regardless of the time spent in treatment, scored higher in values of Conservation than the cocaine users who were not in treatment. Discussion: These results reveal the relationship between attendance on a cocaine-dependence treatment program and personal values, together with their potential importance in treatment and rehabilitation programs for cocaine users.

Key words: cocaine use, cocaine dependence treatment, openness to change values, conservation values. 


\section{INTRODUCCIÓN}

$\mathrm{E}$ alto nivel de consumo de cocaína existente en España - es el país, junto con Estados Unidos, en dónde se da el mayor porcentaje de consumo de esta sustancia de todo el mundo (United Nations Office on Drugs and Crime, 2008) -, que además parece estar en constante aumento (Observatorio Español sobre Drogas [OED], 2007), y los problemas asociados a su consumo, como muertes, accidentes o tráfico de sustancias (OED, 2007), convierten a este fenómeno en un objetivo urgente de estudio (Moral, Ovejero, Sirvent y Rodríguez, 2005). Aunque la mayor parte de las investigaciones que abordan esta cuestión provienen de las ciencias médicas y de la psicología clínica (Caballero, 2005; Llopis, 2001; Lorea, FernándezMontalvo, López-Goñi y Landa, 2009), es importante tener en cuenta también la dimensión psicosocial del uso de sustancias adictivas (Navarro, 2000; Pons, Berjano y García, 1996; Saiz, 2007). En este sentido, una cuestión fundamental son las características diferenciales de los consumidores de cocaína, en cuanto a actitudes, normas subjetivas o valores personales, que pueden estar relacionadas con este consumo (Pedrero, Rojo y Olivar, 2008; Saiz, 2009).

A pesar de que se ha localizado un importante número de factores de riesgo y protección a considerar en los programas preventivos (Becoña, 1999, 2002; Newcomb y Félix-Ortiz, 1992), todavía son necesarias investigaciones que aporten datos que permitan mejorar los programas de tratamiento (López, Becoña, Vieitez, Cancelo, Sobradelo, García, et al., 2008). En este sentido, los valores, como aspectos básicos del individuo que pueden influir en la conducta, dirigiendo su comportamiento y contribuyendo a constituir su estilo de vida (Ros, 2001), podrían desarrollar una función determinante dentro de los distintos programas de tratamiento.

Aunque algunos tratamientos psicosociales para la dependencia de sustancias, como la terapia cognitivo-conductual (Beck, Wright y Newman, 1999; Caballero, 2005) y la terapia psicodinámica (Fonagy, Roth y Higgitt, 2005; Guthrie, Moorey, Margison, Barker, Palmer y McGrath, 1999) no prestan especial atención a los valores, otros programas de tratamiento, como la terapia de aceptación y compromiso (Wilson y Luciano, 2008), el modelo transteórico de Prochaska y DiClemente $(1982,1983)$ y los grupos de autoayuda, (Alcohólicos Anónimos, 2003; Humphreys, Wing, McCarty, Chappel, Gallant y Haberle, 2004; Narcóticos Anónimos, 1991), sí tienen en cuenta el papel de diferentes valores como elementos del proceso de cambio.

Además, algunos autores (Kristiansen, 1985, 1986; Martínez-Sánchez y Ros, 2001; Schwartz e Invar-Saban, 1988) han subrayado el papel de valores en comportamientos de riesgo para la salud. Existen investigaciones que han mostrado la relación de distintos valores, tanto a nivel cultural (Megías, 2000; Saiz, 2007) como a nivel personal (Pons y Berjano, 1999; Rojo, Valdemoro y Cervera, 1994), con el consumo de sustancias adictivas. Así, se han analizado los valores instrumentales y terminales (Rokeach, 1973) que caracterizan a los adictos a la heroína (Rojo, Valdemoro y Cervera, 1994), la relación entre valores convencionales y consumo de drogas ilegales (Liu y Kaplan, 2001), los valores que se detectan como factores de riesgo o factores protectores en el consumo de alcohol y drogas ilegales con la técnica de rejilla (Ruiz, Lozano y Polaina, 1994), o la relación entre los valores del modelo de Schwartz y el consumo de alcohol (Pons y Berjano, 1999; Pons, Berjano y García, 1996).

El presente estudio está centrado en el papel de los valores de Apertura al Cambio y Conservación del modelo de Schwartz (2001) en el tratamiento por dependencia de la cocaína. La teoría de los valores personales elaborada por Schwartz y Bilsky $(1987,1990)$ y revisada posteriormente por Schwartz (2001), considera que los valores deben entenderse como "metas deseables y transituacionales, que varian en importancia y sirven como principios guía en la vida de una persona o de otra entidad social". Schwartz (2001) localizó el origen de los valores en tres necesidades universales para la existencia humana: biológicas, de interacción social coordinada y de supervivencia de los grupos. De estas tres necesidades universales derivó diez tipos motivacionales de valor: poder, logro, hedonismo, estimulación, autodirección, universalismo, benevolencia, tradición, conformidad y seguridad.

Además, la teoría de Schwartz (2001) establece la existencia de una serie de relaciones dinámicas entre estos valores, considerando que algunos son compatibles entre ellos, mientras que otros son incompatibles. Estas relaciones entre los valores conforman una estructura coherente y ordenada que se expresa gráficamente de forma circular $y$, tal como se observa en la figura 1, permite la localización de las relaciones de compatibilidad entre valores cercanos y de conflicto entre los valores opuestos. El modelo considera dos dimensiones bipolares básicas: la primera dimensión se define por Apertura al cambio (que incluye los valores de Autodirección y Estimulación) versus Conservación (que incluye los valores de Tradición, Conformidad y Seguridad); mientras que la segunda dimensión opone Autobeneficio (que incluye Poder y Logro) versus Autotrascendencia (que comprende los valores de Universalismo y Benevolencia). El valor Hedonismo está relacionado tanto con apertura al cambio como con autobeneficio.

La dimensión de valores que se analiza en este estudio, apertura al cambio versus conservación, agrupa los valores en función de si comparten una cierta inclinación al cambio o si se interesan por conservar el status quo. La investigación se centra en estos valores porque han mostrado relación con el consumo de sustancias adictivas en anteriores estudios (Pons y Berjano, 1999; Ruiz, Lozano y Polaino, 1994; Tamayo, Nicaretta y Ribeiro, 1995). Los valores de apertura al cambio enfatizan la independencia de pensamiento y acción y el deseo y apertura a nuevas experiencias. Dentro de estos valores se incluyen: autodirección -definido como pensamiento independiente y elección de la acción, creatividad, exploración-, estimulación -definido como entusiasmo, novedad y cambios en la vida- y hedonismo -entendido como placer y gratificación sensual para uno mismo-. Los valores de conservación enfatizan el orden, la armonía y el autocontrol, incluyendo: tradición -definido como respeto, compromiso y aceptación de las costumbres e ideas que proporcionan la cultura tradicional o la religión-, conformidad -definido como restricción de las acciones, inclinacio- 


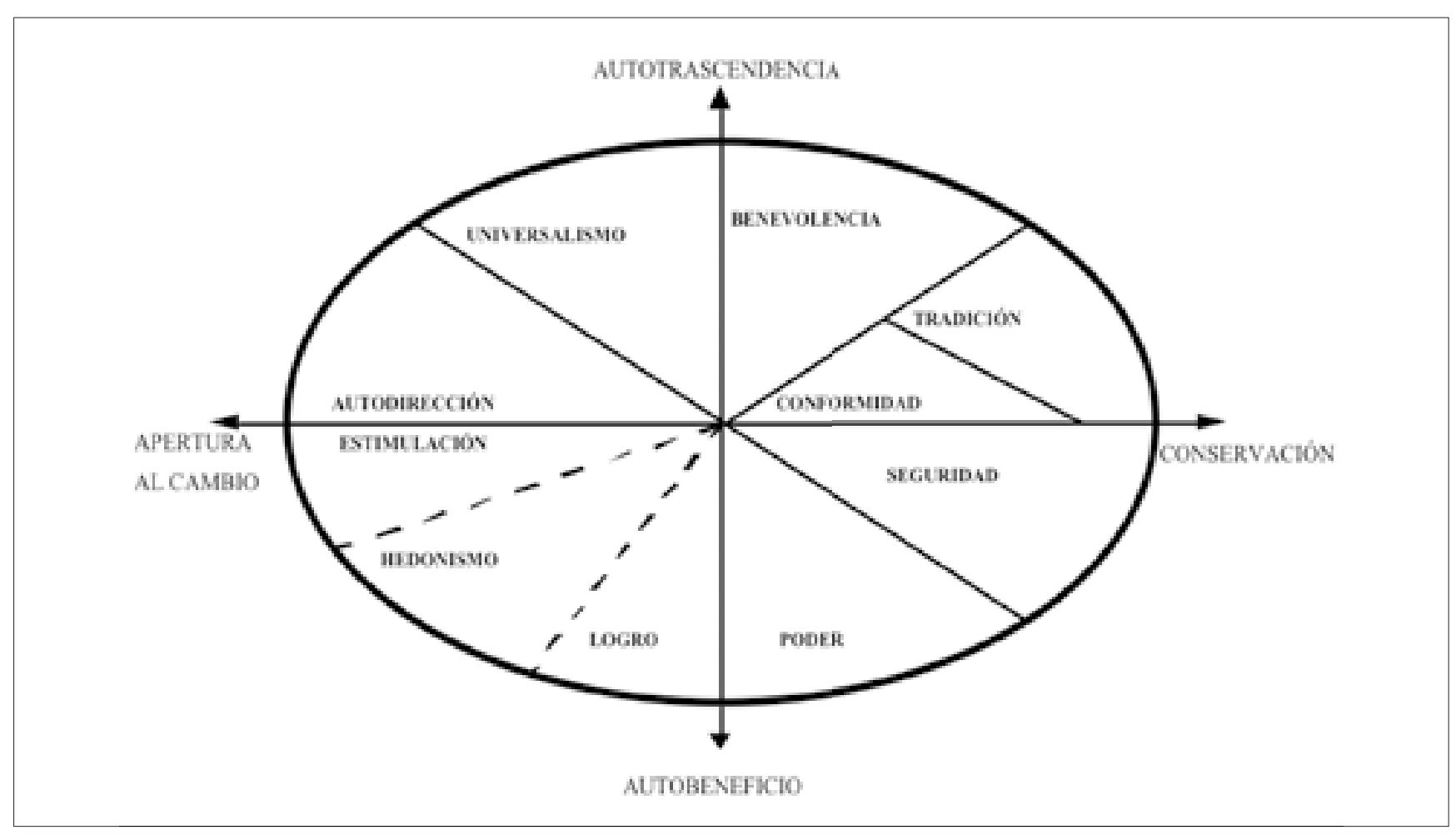

Figura 1. Modelo teórico de las relaciones entre los valores personales agrupados en dimensiones bipolares en función de su similitud (Schwartz, 2001).

nes e impulsos que pudiesen molestar o herir a otros y violar expectativas o normas sociales- y seguridad -que se define como armonía, estabilidad y seguridad en la sociedad, de las relaciones y de sí mismo-.

El presente estudio tiene como objetivo general examinar la relación entre la asistencia a un programa de tratamiento por dependencia de la cocaína y la jerarquía de valores personales de los sujetos consumidores. En concreto se analiza la prioridad que los sujetos que asisten a un programa de tratamiento dan a los valores de conservación y apertura al cambio frente a los que no reciben tratamiento. Teniendo en cuenta los resultados de anteriores estudios, que relacionan el consumo abusivo de alcohol y drogas con el mantenimiento de valores como la búsqueda de placer y estimulación (Pons y Berjano, 1999; Ruiz, Lozano y Polaina, 1994; Tamayo, Nicaretta y Ribeiro, 1995), mientras que el no consumo de sustancias se relaciona con la aceptación de tradiciones, normas y convenciones sociales (Ruiz, Lozano y Polaina, 1994; Tamayo, Nicaretta y Ribeiro, 1995), y dado que los valores de la dimensión apertura al cambio hacen referencia a nuevas emociones y a la búsqueda de placer inmediato, mientras que los valores de la dimensión conservación hacen referencia a un estilo de vida que excluye situaciones estimulantes y de riesgo, y que enfatiza el autocontrol (Schwartz, 2001), se espera que los valores de los consumidores de cocaina que asisten a un programa de tratamiento se diferencien de los consumidores de cocaína que no han recibido tratamiento. De modo que aquellos que asisten a un programa para abandonar su adicción, tiendan a dar menos prioridad a los valores de apertura al cambio y más a los valores de conservación que los sujetos que no se encuentran bajo tratamiento. Puesto que se ha comprobado que la terapia puede ejercer un cambio en los valores de las personas bajo adicciones (Prochaska y Di Clemente, 1982, 1983; Humphreys, et al., 2004; Wilson y Luciano, 2008), se espera que las diferencias entre los sujetos que no asisten a un programa de tratamiento y los que sí lo hacen sean más significativas, en los valores analizados, en la medida que aumente el tiempo que estos últimos llevan asistiendo al programa.

\section{MÉTODOS}

\section{Diseño}

Para probar la hipótesis planteada se empleó un diseño cuasi-experimental, que incluía tres grupos de sujetos en condiciones diferentes. Dos recibieron tratamiento para superar su dependencia a la cocaina durante periodos de tiempo distintos (más de tres meses y menos de tres meses) y uno no recibió ningún tratamiento (grupo control). A todos los grupos se les administró el mismo instrumento de medición de valores personales. Aunque, no se trata de un estudio experimental, puesto que no se hace una asignación aleatoria de los sujetos a las tres condiciones de la investigación, ni tampoco de un diseño longitudinal ya que no se miden los mismos sujetos en distintos momentos temporales, el hecho de que éste sea un estudio cuasi-experimental ofrece mayores garantías de validez interna que un estudio no-experimental (Cook y Campbell, 1979; Pascual, Frias y Garcia, 1997). Además, hay que tener en cuenta que los estudios experimen- 
tales y los longitudinales o de medidas repetidas presentan también algunos inconvenientes, como las cuestiones éticas y prácticas en el caso de los diseños experimentales, que no permitirian realizar una aleatorización de los sujetos en el presente estudio, o el aumento del error tipo I y la posibilidad de que existan efectos de variables extrañas en los diseños longitudinales (García, Frías y Pascual,1999; Pascual, Frías y García, 1997) que en el caso del estudio actual, a pesar de poder constatar un cambio en los sujetos, no permitirian eliminar la posibilidad de que el cambio se debiese a otras variables diferentes a la terapia.

Sin embargo, es necesario considerar que, en el presente estudio, aunque se constate la diferencia que se plantea en la hipótesis entre los valores de los sujetos en función de si asisten o no a un programa de tratamiento, no se puede descartar que existan otras causas de la variación diferentes a la asistencia al tratamiento, si bien los supuestos teóricos que describe la literatura sobre el tema apoyarian la hipótesis del papel de la terapia en estas diferencias (Pérez, 2008).

\section{Participantes}

El presente estudio se realizó con 411 adultos consumidores de cocaína residentes en la Comunidad de Madrid. Aproximadamente la mitad de estos sujetos (52,8 \%) no se encontraba bajo ningún tratamiento por consumo de cocaína, un 19,2\% había iniciado el tratamiento hacía menos de 3 meses, y el 28,0\% superaba los tres meses de tratamiento. La muestra estaba compuesta por 121 mujeres $(29,4 \%)$ y 290 hombres (70.6 \%). La edad de los sujetos se localizaba entre los 19 y los 45 años $(M=27,83, D T=6,55)$.

\section{Procedimiento}

El grupo de consumidores de cocaína sin tratamiento se reunió mediante la estrategia de "contactos en cadena", en donde a partir de un consumidor se accede por referencias a otros. Además, se solicitaron participantes para el estudio en la prensa (20 Minutos de Madrid y Gaceta Universitaria de la UCM) y mediante la colocación de carteles y distribución de volantes en zonas estratégicas (alrededor de 2.000 carteles y 300 volantes). Mientras que el grupo de consumidores de cocaína en tratamiento está formado por sujetos que acudian a distintos Centros de Atención Integral a Drogodependientes (CAID) y a otros centros para la rehabilitación de la Comunidad de Madrid (públicos y privados)' 1 .

La administración de las pruebas a los consumidores en tratamiento se realizó de manera colectiva por personal entrenado. En cambio, dada la imposibilidad de establecer contacto físico con buena parte de las personas pertenecientes al grupo de consumidores sin tratamiento, se les hizo

${ }^{1}$ CAIC del Hospital San Juan de Dios, CAID de Alcalá de Henares, CAID de Majadahonda, CAID de Móstoles, CAID de Usera, Centro de Tratamiento El Olivar, Comunidad Terapéutica Dianova, Comunidad Terapéutica Narconón, Federación de Alcohólicos de la Comunidad de Madrid, Narcóticos Anónimos y Proyecto Hombre. llegar el cuestionario por medio de informadores clave o se les remitió el mismo por correo postal, con franqueo pagado, o por correo electrónico. La condición de anonimato fue garantizada por escrito en todos los casos.

\section{Instrumento}

Para medir los valores de apertura al cambio (autodirección, estimulación y hedonismo) y conservación (tradición, conformidad y seguridad) se utilizó el "Portrait Values Questionnaire" PVQ (Schwartz, Melech, Lehmann, Burgess, Harris y Owens, 2001; Schwartz, 2003) basado en el modelo teórico de Schwartz (2001). De este instrumento se extrajeron los 17 ítems que miden apertura al cambio y conservación, los cuales consisten en una frase descriptiva que los sujetos tienen que valorar con una escala cuyo rango va de 1 (no se parece nada a mi) a 6 (se parece mucho a mí). En la tabla 1 se recogen los ítems con los que se mide cada uno de los valores, el valor autodirección está medido mediante 3 ítems, estimulación mediante 2 ítems, hedonismo mediante 2 ítems, tradición mediante 4 items, conformidad mediante 3 items y seguridad mediante 3 items. Finalmente, estos seis valores utilizados en el estudio fueron calculados promediando las respuestas de los ítems que miden cada valor.

EI PVO está basado en el "Schwartz Value Survey" SVS (Schwartz, 2001), el cual ha sido aplicado en numerosas ocasiones (por ej., Martínez y Garcia, 2008; Ros y Gouveia, 2001), mostrando una alta validez transcultural (Schwartz, Verkasalo, Antonovsky y Sagiv, 1997; Spini, 2003). Ha sido probado en más de 200 muestras en más de 60 paises (Schwartz, 1992; Schwartz y Sagiv, 1995) y utilizado para explicar un amplio rango de actitudes y comportamientos en distintos países (Schwartz y Bardi, 2001). El PVO reduce el número de ítems del SVS de 56 a 29 y su contestación resulta más sencilla. La versión española del PVQ ha sido validada por Saiz (2008) y Zoblina (2004) y puede consultarse, también, en el trabajo de Gómez, Gaviria y Fernández (2006). Los coeficientes de confiabilidad para cada uno de los valores fueron los siguientes: autodirección, 0,45; estimulación, 0,66 ; hedonismo, 0,68; conformidad, 0,51; tradición 0,45; y seguridad 0,52. Estos coeficientes se encuentran dentro del rango de variación comúnmente observado en estos tipos de valores (por ej., Martínez y García, 2007; Roccas, Sagiv, Schwartz, y Knafo, 2002).

\section{Resultados}

Para analizar la relación entre la asistencia a un programa de tratamiento y los valores en adultos consumidores de cocaina, teniendo en cuenta el posible efecto del género de los sujetos, se realizó un MANOVA factorial $3 \times 2$ entre el tiempo de tratamiento (sin tratamiento, menos de tres meses y más de tres meses) y el género (masculino y femenino) con los valores de apertura al cambio (autodirección, estimulación y hedonismo) y conservación (seguridad, tradición y conformidad) de Schwartz en estos sujetos. El análisis mostraba que únicamente el efecto principal del tiempo de tratamiento fue 
Tabla 1. Ítems del Cuestionario de Valores PVQ empleados

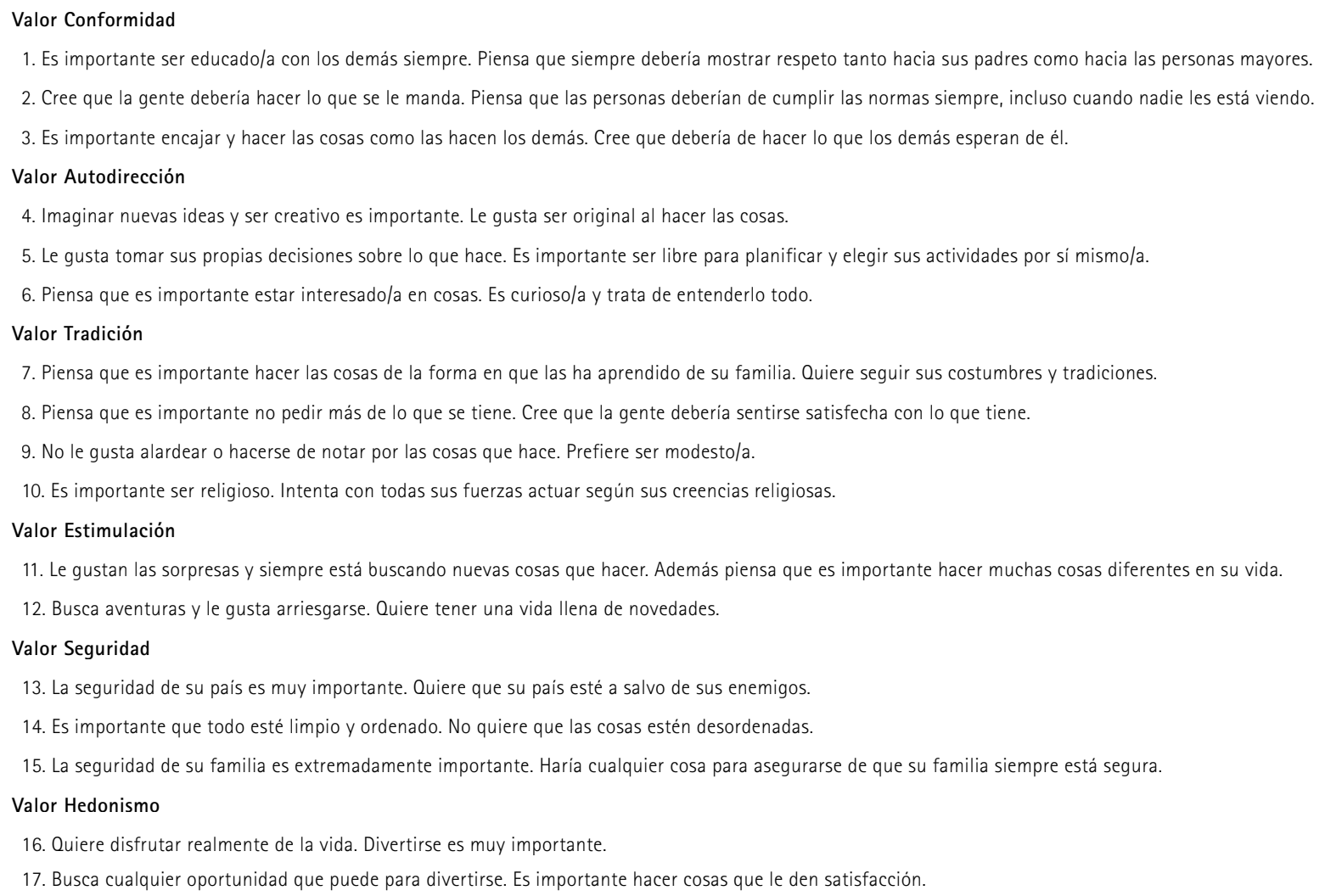

significativo $\left(\Lambda=0,813, F_{12,800}=7,27 ; p<0,001\right)$. Ni el género de los sujetos ni su interacción con el tiempo de tratamiento resultaron estadísticamente significativos en el análisis $(p>$ $0,05)$, mostrando así que la relación entre el tratamiento por consumo de cocaína y los valores de los sujetos no se ve influida por el hecho de que éstos sean hombres o mujeres.

Por lo tanto, seguidamente se analizaron los efectos principales univariados del tiempo de tratamiento en los valores de apertura al cambio y de conservación. Como puede observarse en la tabla 2, el efecto del tiempo de tratamiento es significativo en todos los valores analizados salvo en auto- dirección $(p>0,05)$. En los otros dos valores de apertura al cambio (estimulación y hedonismo) se observa que el grupo que lleva más de tres meses de tratamiento se diferencia del grupo sin tratamiento, puntuando significativamente menos en estos valores, mientras que el grupo que lleva menos de tres meses de tratamiento no se diferencia de ninguno de los otros dos grupos de sujetos en estos valores. Por otra parte se comprueba que en todos los valores de conservación (seguridad, tradición y conformidad), los sujetos en tratamiento, tanto durante menos de tres meses como durante más de tres meses, dan más prioridad a estos valores que los sujetos adictos a la cocaína que no han recibido tratamiento.

Tabla 2. Análisis de la Varianza de los Efectos Univariados entre el tiempo de tratamiento por consumo de cocaína y los valores de Apertura al cambio y Conservación.

\begin{tabular}{|c|c|c|c|c|c|}
\hline & Sin tratamiento $(\mathrm{N}=217)$ & Menos de 3 meses $(N=79)$ & Más de 3 meses $(\mathrm{N}=115)$ & $F(2,405)$ & $\mathrm{p}$ \\
\hline \multicolumn{6}{|l|}{ Apertura al cambio } \\
\hline Autodirección & $5,20(0,61)$ & $4,97(0,76)$ & $4,94(0,82)$ & 1,68 & 0,188 \\
\hline Estimulación & $4,90(0,93)^{1}$ & $4,57(1,12)$ & $4,32(1,17)^{2}$ & 6,19 & 0,002 \\
\hline Hedonismo & $5,18(0,78)^{1}$ & $4,95(1,01)$ & $4,86(0,97)^{2}$ & 6,49 & 0,002 \\
\hline \multicolumn{6}{|l|}{ Conservación } \\
\hline Conformidad & $2,68(1,08)^{2}$ & $3,52(1,23)^{1}$ & $3,48(1,32)^{1}$ & 12,25 & $<0,001$ \\
\hline Tradición & $3,12(0,79)^{2}$ & $3,67(1,00)^{1}$ & $3,64(0,93)^{1}$ & 12,79 & $<0,001$ \\
\hline Seguridad & $3,51(1,25)^{2}$ & $4,56(1,12)^{1}$ & $4,49(1,09)^{1}$ & 26,76 & $<0,001$ \\
\hline
\end{tabular}




\section{DISCUSIÓN}

Los resultados confirman la relación entre el tratamiento por consumo de cocaína y los valores de los sujetos consumidores. En primer lugar, se observa que tal y como se estableció en las hipótesis, los resultados ponen de manifiesto una tendencia a que las personas den menos prioridad a los valores de apertura al cambio en la medida en que se encuentran asistiendo a un programa de tratamiento por consumo de cocaina: se comprueba que las personas consumidoras de cocaína que llevan más de tres meses de tratamiento conceden menos importancia a los valores de estimulación y hedonismo que los sujetos que no se encuentran bajo tratamiento. De modo que aquellas personas que inician y perseveran en un proceso de rehabilitación no perseguirian la estimulación y el hedonismo, como metas deseables, con la misma intensidad que aquellas personas que no han iniciado un programa de tratamiento. El hecho de que los sujetos que han comenzado el tratamiento hace menos de tres meses no se diferencien de los sujetos que no siguen ningún tratamiento, podria estar indicando la existencia de un cambio en la prioridad que se concede a estos valores, que se produciría de manera progresiva a medida que avanza el tiempo de tratamiento. Por otra parte, la circunstancia de que no se observen diferencias en la prioridad que se le da al valor autodirección entre los consumidores de cocaina, en función de si se encuentran o no bajo tratamiento, podría estar relacionado con que este valor implica el planteamiento de objetivos y la persistencia hasta la consecución de los mismos (Schwartz, 2001), y es posible que sea un valor deseable para un exconsumidor en tratamiento, ya que le ayudaría a persistir en la abstinencia aunque en el entorno tropiece con influencias que le inviten a la recaída.

También se confirma la mayor prioridad que los consumidores de cocaina que se encuentran bajo tratamiento dan a los valores de conservación. Tanto los consumidores que llevan más de tres meses como los que llevan menos de tres meses de tratamiento dan más importancia a los valores de conformidad, tradición y seguridad que las personas que no se encuentran bajo tratamiento. Se podría deducir así que, al ingresar en un programa de tratamiento, la persona otorga una mayor puntuación a aquellos valores que hacen énfasis en buscar la seguridad y en una vida acorde con las normas socio-culturales existentes. La estabilización en los valores de esta dimensión encontrados después de los tres meses de tratamiento podria corresponderse con un momento de cambio y concienciación sobre la dependencia a la cocaína, que Prochaska, DiClemente y Norcross (1992) y Prochaska, Velicer, Rossi, Goldstein, Marcus y Rakowski (1994) denominaron etapa de "mantenimiento". Es decir, llegado el momento en el que el sujeto en tratamiento logra la modificación de una importante sección de su jerarquía de valores, este cambio se podria mantener en el tiempo gracias a las consecuencias positivas que implica.

En definitiva, se confirman diferencias en los valores personales de los consumidores de cocaína en función de si se encuentran o no bajo tratamiento, revelando que las personas bajo tratamiento tienden a dar menor prioridad a los valores de apertura al cambio (hedonismo y estimulación) y mayor prioridad a los valores de conservación (conformidad, tradición y seguridad) que las personas que no se encuentran bajo tratamiento. Esto se halla en línea con los resultados de estudios anteriores, que muestran que el consumo de sustancias se relaciona positivamente con el mantenimiento de valores que suponen la búsqueda del placer y la estimulación, y negativamente con la aceptación de las normas y tradiciones sociales (Liu y Kaplan, 2001; Pons y Berjano, 1999).-

Por otra parte, de los resultados de este estudio se pueden derivar, de forma tentativa, algunas implicaciones prácticas. Así, con el fin de facilitar el proceso de rehabilitación de la dependencia a la cocaína, resultaría positivo prestar mayor atención a la jerarquía de valores del sujeto y otorgarles una mayor importancia en el desarrollo de este proceso. Además, resultaría oportuno insistir en el empleo de estos elementos dentro de los distintos programas de prevención. No obstante, para una adecuada comprensión del problema que permita intervenir de manera efectiva sobre éste, será necesaria una lectura global e integradora del mismo que reconozca, en todo momento, el carácter multifactorial del consumo de sustancias y la necesidad de una intervención multidisciplinar (Valverde, 1996).

Finalmente, con respecto a la metodología empleada hay que tener en cuenta que dado que el presente estudio es cuasi-experimental, cabe la posibilidad de que en lugar de que se produzca una modificación en la jerarquía de valores de los sujetos que asisten a un programa de tratamiento, según sugiere la literatura sobre esta cuestión, sean las personas con mayores puntuaciones en valores de conservación y menores en valores de apertura al cambio las que inicien y perseveren en los programas de tratamiento. Por tanto, aunque los resultados obtenidos son los que se prevén en las hipótesis no se pueden descartar otras explicaciones alternativas a este mismo patrón de resultados. La realización de un estudio experimental, en el que se aplicase la asignación aleatoria en la formación de grupos o de un estudio longitudinal en el que se midiesen los mismos sujetos en diferentes momentos, ayudaría a confirmar esta cuestión, si bien plantearía otros problemas metodológicos comentados anteriormente. Sin embargo, como una primera aproximación al problema, el estudio pone de manifiesto la relación entre el tratamiento por consumo de cocaina y los valores de los sujetos consumidores; y por lo tanto, la importancia de los valores personales en el tratamiento y la rehabilitación de la persona dependiente de la cocaína.

\section{REFERENCIAS}

Alcohólicos Anónimos. (2003). Alcohólicos anónimos: el libro grande en línea. Recuperado el 12 de noviembre de 2007 de http:// www.aa.org/bigbookonline/sp_tableofent.cfm.

Beck, A., Wright, F. y Newman, C. (1999). Terapia cognitiva de las drogodependencias. Barcelona: Paidós.

Becoña, E. (1999). Bases teóricas que sustentan los programas de prevención de drogas. Madrid: PNSD. 
Becoña, E. (2002). Bases científicas de la prevención de las drogodependencias. Madrid: PNSD.

Caballero, L. (2005). Adicción a cocaina: neurobiología clínica, diagnóstico y tratamiento. Madrid: PNSD.

Cook, T. D. y Campbell, D. T. (1979). Quasi-experimentation: Design and analysis issues for field settings. Chicago: Rand McNally.

Fonagy, P., Roth, A. y Higgitt, A. (2005). The outcome of psychodynamic psychotherapy for psychological disorders. Clinical Neuroscience Research, 4, 367-377.

Garcia, J. F., Frías, M. D. y Pascual, J. (1999). Los diseños de la investigación experimental: Comprobación de las hipótesis. Valencia: Cristóbal Serrano Villalba.

Gómez, A., Gaviria, E. y Fernández, I. (2006). Psicología social. Madrid: Sanz \& Torres.

Guthrie, E., Moorey, J., Margison, F., Barker, H., Palmer, S. y McGrath, G. (1999). Cost-effectiveness of brief psychodynamic-interpersonal therapy in high utilizers of psychiatric services. Archives of General Psychiatry, 56, 519-526.

Humphreys, K., Wing, S., McCarty, D., Chappel, J., Gallant, L. y Haberle, B. (2004). Self-help organizations for alcohol and drugs problems: toward evidence-based practice and policy. Journal of Substance Abuse Treatment, 26, 151-158.

Kristiansen, C. (1985). Value correlates of preventive health behavior. Journal of Personality and Social Psychology, 49, 748-758.

Kristiansen, C. (1986). A two-value model of preventive health behavior. Basic and Applied Social Psychology, 7, 173-183.

Liu, X. y Kaplan, H. (2001). Role strain and illicit drug use: the moderating influence of commitment to conventional values. Journal of Drug Issues, 31, 833-856.

Llopis, J. (2001). Dependencia, intoxicación aguda y sindrome de abstinencia por cocaina. Adicciones, 13, 147-166.

López, A., Becoña, E., Vieitez, I., Cancelo, J., Sobradelo, J., García, J. M. et al. (2008). ¿Qué ocurre a los 24 meses del inicio del tratamiento en una muestra de personas con dependencia de la cocaina? Adicciones, 20, 347-356.

Lorea, I., Fernández-Montalvo, J., López-Goñi, J. J. y Landa, N. (2009). Adicción a la cocaina y trastornos de personalidad: un estudio con el MCMI-II. Adicciones, 21, 57-63.

Martinez, I. y Garcia, J. F. (2007). Impact of parenting styles on adolescents' self-esteem and internalization of values in Spain. The Spanish Journal of Psychology, 10, 338-348.

Martínez, I. y García, J. F. (2008). Internalization of values and self-esteem among Brazilian teenagers from authoritative, indulgent, authoritarian, and neglectful homes. Adolescence, 43, 13-29.

Martínez-Sánchez, E. y Ros, M. (2001). Los significados de la salud y la salud como valor. En M. Ros y V. V. Gouveia (Eds.). Psicología social de los valores humanos. Madrid: Biblioteca Nueva.

Megías, E. (Dir.) (2000). Los valores de la sociedad española y su relación con las drogas. Barcelona: Fundación la Caixa.

Moral, M., Ovejero, A., Sirvent, C. y Rodríguez, F., (2005). Prevención e intervención psicosocial sobre el consumo juvenil de sustancias psicoactivas: Comparación de la eficacia preventiva de cuatro programas. Intervención Psicosocial, 14, 198-208.

Narcóticos Anónimos. (1991). Narcóticos Anónimos. Chatsworth [California]: Narcotics Anonymous World Services.

Navarro, J. (2000). Factores de riesgo y protección de carácter social relacionados con el consumo de drogas. Madrid: Ayuntamiento de Madrid.
Newcomb, M. y Félix-Ortiz, M. (1992). Multiple protective and risk factors for drug use and abuse: cross-sectional and prospective findings. Journal of Personality and Social Psychology, 63, 280296.

Observatorio Español sobre Drogas. (2007). Informe 2007. Madrid: Ministerio del Interior.

Pascual, J., Frias, D. y Garcia, F. (1997). Manual de psicologia experimental: Metodología de investigación. Barcelona, Spain: Ariel.

Pedrero, E., Rojo, G. y Olivar, A. (2008). Valores sociales y adicción: aplicabilidad y propiedades psicométricas del cuestionario VAL89. Adicciones, 20,327-336.

Pérez, J. F. G. (2008). Métodos de investigación, diseño y técnicas en las ciencias del comportamiento. Valencia, Spain: Palmero Ediciones.

Pons, J. y Berjano, E. (1999). El consumo abusivo de alcohol en la adolescencia: un modelo explicativo desde la psicología social. Madrid: PNSD.

Pons, J., Berjano, E. y García, F. (1996). Variables psicosociales que discriminan el consumo abusivo de alcohol en la adolescencia. Adicciones, 8, 177-191.

Prochaska, J. y DiClemente, C. (1982). Transtheoretical therapy: toward a more integrative model of change. Psychotherapy: Theory, Research and Practice, 19, 276-288

Prochaska, J. y DiClemente, C. (1983). Stages and processes of selfchange of smoking: toward an integrative model of change. Journal of Consulting and Clinical Psychology, 51, 390-395.

Prochaska, J., DiClemente, C. y Norcross, J. (1992). In search of how people change: applications to addictive behaviors. American Psychologist, 47, 1102-1114.

Prochaska, J., Velicer, W., Rossi, J., Goldstein, M., Marcus, B., Rakowski, W. et al. (1994). Stages of change and decisional balance for 12 problem behaviors. Health Psychology, 13, 39-46.

Roccas, S., Sagiv, L., Schwartz, S. y Knafo, A. (2002). The big five personality factors and personal values. Personality and Social Psychology Bulletin, 28, 789-801.

Rojo, M. J., Valdemoro, G. C. y Cervera, G. (1994). Análisis de los valores en toxicómanos, medidos mediante la escala de valores de Rokeach. Anales de Psiquiatría, 10, 272-278.

Rokeach, M. (1973). The nature of human values. New York: The Free Press.

Ros, M. (2001). Valores, actitudes y comportamiento: una nueva visita a un tema clásico. En M. Ros y V. V. Gouveia (Coords.). Psicología social de los valores humanos. Madrid: Biblioteca Nueva.

Ros, M. y Gouveia, V. V. (2001). Validez de los modelos transculturales sobre valores. En M. Ros y V. V. Gouveia (Coords.). Psicología social de los valores humanos. Madrid: Biblioteca Nueva.

Ruiz, P., Lozano, E. y Polaino, A. (1994). Los valores en el adolescente y el consumo de sustancias. Anales de Psiquiatría, 10, 115-120.

Saiz, J. (2007). El abuso de cocaina, ¿Problema de oferta o de demanda social?: un estudio transcultural y correlacional que compara variables macrosociales, económicas y culturales. Adicciones, $19,35-44$.

Saiz, J. (2008). Un estudio del consumo de cocaina en la Comunidad de Madrid desde la psicología social. Tesis Doctoral en Microficha. Madrid: Servicio de Publicaciones de la Universidad Complutense de Madrid. 
Saiz, J. (2009). Estudio empírico de las variables de la Teoría de la Conducta Planificada como factores de riesgo para el consumo de cocaina en tres grupos diferentes, Adicciones, 21, 187-194.

Schwartz, S. (2003). A proposal for measuring value orientations across nations. En European Social Survey (Ed.). Questionnaire development report. Recuperado el 07 de febrero de 2009 de http://www.europeansocialsurvey.org

Schwartz, S. (2001). ¿Existen aspectos universales en la estructura y contenido de los valores humanos? En M. Ros y V. V. Gouveia (Coords.). Psicología social de los valores humanos. Madrid: Biblioteca Nueva.

Schwartz, S. (1992). Universals in the content and structure of values: Theoretical advances and empirical tests in 20 countries. En M. P. Zanna (Ed.), Advances in experimental social psychology (pp. Vol. 25, 1-65). New York: Academic Press.

Schwartz, S. y Bardi, A. (2001). Value hierarchies across cultures. Journal of Cross-Cultural Psychology, 32, 268-290.

Schwartz, S. y Bilsky, W. (1987). Toward a universal psychological structure of human values. Journal of Personality and Social Psychology, 53, 550-562.

Schwartz, S. y Bilsky, W. (1990). Toward a theory of the universal content and structure of values: extensions and cross-cultural replications. Journal of Personality and Social Psychology, 58, 878-891.

Schwartz, S., Melech, G., Lehmann, A., Burgess, S., Harris, M. y Owens, V. (2001). Extending the cross-cultural validity of the theory of basic values with a different method of measurement. Journal of Cross-Cultural Psychology, 32, 519-542.

Schwartz, S. e Invar-Saban, N. (1988). Value self-confrontation as a method to aid to weight loss. Journal of Personality and Social Psychology, 54, 396-404.

Schwartz, S. y Sagiv, L. (1995). Identifying culture-specifics in the content and structure of values. Journal of Cross-Cultural Psychology, 26, 92-116.

Schwartz, S., Verkasalo, M., Antonovsky, A. y Sagiv, L. (1997). Values priorities and social desirability: much substance, some style. British Journal of Social Psychology, 36, 3-18.

Spini, D. (2003). Measurement equivalence of 10 value types from the Schwartz value survey across 21 countries. Journal of CrossCultural Psychology, 34, 3-23.

Tamayo, A., Nicaretta, M. y Ribeiro, R. (1995). Values priorities and drug addiction. Acta Psiquiátrica y Psicológica de América Latina, 41, 300-307.

United Nations Office on Drugs and Crime. (2008). World Drug Report 2008. Recuperado el 10 de febrero de 2009 de http://www. unodc.org.

Valverde, J. (1996). Vivir con la droga. Madrid: Pirámide.

Wilson, K. y Luciano, M. C. (2008). Terapia de aceptación y compromiso: un tratamiento conductual orientado a los valores. Madrid: Pirámide.

Zlobina, A. (2004). Estereotipos nacionales y regionales en Europa y España. En D. Páez, I. Fernández, S. Ubillos, E. y Zubieta (Eds.). Psicología social, cultura y educación. Madrid: Prentice Hall 